\title{
A COMPARISON OF THE EFFICACY AND SAFETY OF ORAL ANTI-DIABETIC DRUGS IN CAUCASIANS AND SOUTH ASIANS: A SYSTEMATIC REVIEW AND META-ANALYSIS
}

\author{
Aoife McMullen, Catriona Skarnes \& Martha Richardson \\ Faculty of Biological Sciences, University of Leeds, UK \\ correspondence to um15agm@leeds.ac.uk
}

Cite as: McMullen A, Skarnes C \& Richardson M. (2020) A comparison of the efficacy and safety of oral anti-diabetic drugs in Caucasians and South Asians: A systematic review and meta-analysis. The Physician 6(1)c10 DOI: 10.38192/1.6.1.c10

\section{Background:}

Type 2 diabetes mellitus is more prevalent, and presents differently, in South Asians than Caucasians. This suggests a possible difference in the genetic disposition of South Asians for this condition. Such genetic difference could also confer a difference in the response of patients of different ethnicities to oral antidiabetic drugs (OADs).

Methods:

This systematic review, with meta-analysis, compares the efficacy and safety of OADs in Caucasian and South Asian type 2 diabetics, using randomised trials published until February 2019. A systematic search of PubMed, Web of Science, Ovid and Scopus databases was performed. 27 studies met the inclusion criteria, with nine eligible for meta-analysis. Data on the change in \% glycosylated haemoglobin (HbA1c) and adverse events (AEs) was studied.

\section{Results:}

This review is limited by small number of included studies, which were often of poor quality. A significant difference in efficacy was found in only one drug. However, trends were noted with other drug classes, but the volume of data was insufficient to draw statistically test. Similarly, there was little data relating to AEs, although some trends were again noted.

Conclusions:

Although the results were disappointing, this study has revealed that research is often presented in a way which prevents race-specific outcomes from being measured. This paper concludes by making recommendations which would enable larger studies to be conducted, potentially finding correlations between ethnicity and drug-related outcomes. This could in turn inform prescribing, allowing patients to be given medications which are more likely to be effective, and less likely to cause them harm. 